\title{
L'impact du Brexit sur la relation franco-allemande
}

\author{
Jean-Marc Trouille ${ }^{1}$ \\ j.m.I.trouille@bradford.ac.uk
}

\section{Introduction}

Le Brexit représente le changement le plus important dans les relations que le Royaume-Uni a entretenu avec l'Europe et le monde depuis la fin de la Seconde Guerre mondiale. La décision britannique de mettre fin à plus de quatre décennies de participation au projet européen, la détermination du gouvernement de Theresa May à extraire son pays de l'Union Européenne (UE), mais aussi de l'Union douanière, du Marché intérieur, de la juridiction de la Cour Européenne de Justice, de l'ensemble des règlementations européennes, et même de la Convention Européenne des Droits de l'Homme, ont des implications multiples et lourdes de conséquences dans de vastes domaines. Le Royaume-Uni est certes le premier pays affecté, et ce sur tous les plans. Toutefois, la France et l'Allemagne, ainsi que le projet européen, sont aussi directement concernés par ce divorce qui laisse présager d'importantes répercussions économiques et politiques, mais aussi un déclin progressif de leur voisin d'outre-Manche, avec les conséquences qui pourront en découler.

Si le Royaume-Uni s'apprête à quitter l'Union, il se prépare de façon extrêmement désordonnée, et dans un climat de profonds clivages politiques et sociaux que la perspective du Brexit ne fait chaque jour qu'aggraver davantage. Il le fait de surcroît dans un contexte international de plus en plus instable et imprévisible, où les grands équilibres géopolitiques fondamentaux établis dans l'après-guerre sont remis en cause, où les valeurs démocratiques sont menacées, et où les sociétés sont de plus en plus divisées. Les valeurs traditionnellement représentées par les démocraties occidentales et les institutions qui les défendent sont chaque jour davantage érodées. Nationalisme économique, nationalisme tout court, souverainisme, protectionnisme, populisme et questions identitaires sont dans l'air du temps, un temps qui n'est pas sans rappeler celui des années 1930. Gouvernements, institutions globales et coopérations internationales sont fragilisés par un nouvel environnement qui leur est de plus en plus hostile et remet en question des principes aussi fondamentaux que la paix, la prospérité, l'ouverture, la libre circulation, et les valeurs libérales des sociétés occidentales.

Face au déclin annoncé que s'inflige la troisième puissance économique européenne et sixième mondiale ${ }^{2}$ dans ce contexte international aussi volatile qu'incertain, quelles perspectives se profilent pour la France et l'Allemagne dans leur relation, leur rapport de force, et à quels défis doivent-elles faire face en Europe, finalité de l'entente francoallemande?

\footnotetext{
${ }^{1}$ Jean-Marc Trouille est Professeur Jean Monnet en intégration économique européenne à l’Ecole de Management de l'Université de Bradford (UK). Ses intérêts de recherche portent sur les relations économiques franco-allemandes, les politiques européennes, et plus récemment, sur les questions de développement et d'intégration régionale en Afrique dans une perspective comparative. II dirige depuis 2016 le Réseau Jean Monnet "The European Union, Africa and China in the Global Age" (EU-EAC).

${ }^{2}$ Le Royaume-Uni a été détrôné en 2018 de son 5e rang par l'Inde, ancient joyau de son Empire colonial.
} 
Le présent chapitre examine le rôle et l'importance du Royaume-Uni dans la relation francoallemande, la signification du Brexit, son impact sur le rapport de forces entre Paris et Berlin, et les défis que soulève le divorce anglo-européen auxquels l'Allemagne et la France vont devoir faire face. Quelle fonction le Royaume-Uni a-t-il traditionnellement joué dans le jeu complexe des trois puissances économiques européennes? Comment expliquer qu'un pays qui ait tant apporté à l'Europe, et qui ait tant bénéficié de son appartenance à l'UE, puisse "déclarer forfait" et quitter l'Union, peut-être même, en l'absence d'un accord, en claquant la porte? A quoi pourrait ressembler un Royaume-Uni post-Brexit? Pourrait-il être tenté d'adopter des politiques économiques non coopératives à l'égard de ses voisins continentaux? Dans quelle mesure le rapport de forces entre Paris et Berlin sera-t-il affecté par ce divorce au sein de la famille européenne? Et en fin de compte, comment l'entente franco-allemande pourra-t-elle surmonter le défi du Brexit?

\section{Le Royaume-Uni dans la relation franco-allemande}

Les relations d'après-guerre entre les trois principales puissances européennes ont fréquemment été sources de tensions. L'attitude britannique vis-à-vis de l'Europe a traditionnellement consisté à empêcher la formation d'alliances sur le continent. La France a, en revanche, souvent adopté une stratégie visant à isoler la Grande Bretagne afin de ne pas se retrouver elle-même dans une telle position, tandis que l'Allemagne, puissance de par sa taille économique et démographique mais peu encline à assumer seule un leadership européen, a naturellement recherché une entente bilatérale avec la France. Un partenariat tripartite pour l'Europe (Maclean et Trouille 2001), bien que souhaitable, se serait avéré trop complexe à gérer. Comme le soulignait pertinemment Otto von Bismarck sur le principe même de toute alliance tripartite: "Dans l'interaction de trois pouvoirs, je préfère être l'un des deux." ${ }^{3}$ Ainsi l'attitude britannique vis-à-vis de la relation franco-allemande a pu être comparée à celle d'une maîtresse délaissée, dont l'amant présumé manifestait sa préférence pour l'union légitime avec Germania, sacralisée par le Traité de l'Elysée. Dès qu'une divergence entre Paris et Berlin se fait jour, certains observateurs outre-Manche sont prompts à prédire la fin du couple franco-allemand, voire de l'Europe. Dans ses Mémoires (Thatcher 1995), Margaret Thatcher ne dissimulait guère son agacement face au fait que la scène européenne était dominée de facto par un axe entre Paris et Bonn, ni son hostilité à une Europe "carolingienne" que construisaient Français et Allemands "en se dupant les uns les autres". Lorsqu'au début des années 1990 François Mitterrand, après avoir échoué dans ses tentatives de ralentir le processus d'unification allemande, se décida à le soutenir, Mme Thatcher, irritée, alla jusqu'à le comparer au Maréchal Pétain, l'accusant implicitement de collaboration avec l'ennemi. De telles réactions sont certes excessives, mais loin d'être exceptionnelles. L'idée selon laquelle les Britanniques ont contribué à libérer la France contre le nazisme pour la voir ensuite "pactiser" avec l'ennemi d'antan a encore de beaux jours outre-Manche.

Dans ses mémoires, François Mitterrand exprimait sa déception que l'entente cordiale franco-britannique ait toujours été limitée à des déclarations courtoises (la comparaison avec le Maréchal mise à part), mais dénuées de substance et d'engagement réciproque, ne permettant guère d'envisager une alternative crédible à la relation franco-allemande en Europe. II écrivait notamment: "Elle (Mme Thatcher) avait alors, avec passion, dressé le tableau d'une Europe dominée par la puissance allemande et m'avait prié d'examiner les conditions d'un rapprochement franco-britannique capable de la contrebalancer. Je lui avais répondu, comme à chaque fois que nos conversations abordaient ce sujet, qu'à mon vif regret l'alliance entre nos deux pays, toujours célébrée dans les discours officiels, n'avait

\footnotetext{
${ }^{3}$ Cité par Braunberger, G. 'Allianzübungen in der Dreiecksdiplomatie', Frankfurter Allgemeine Zeitung, 15 octobre 1998, p17.
} 
guère de contenu, que nous ne parvenions jamais, du fait de son pays, à conclure un accord particulier sur quelque objet que ce fût, y compris pour harmoniser les systèmes d'armement les plus simples, que la Grande-Bretagne n'avait jamais de marge d'action suffisante pour échapper au contrôle des Etats-Unis, et que je n'échangerais pas la construction européenne, dont l'Allemagne fédérale constituait l'un des piliers, contre une entente francoanglaise souhaitable, mais réduite aux bons sentiments" (Mitterrand 1996). La conception britannique traditionnelle des relations européennes, héritée des siècles passés, était restée fondée sur la recherche d'équilibres et d'alliances d'Etat à Etat et sur la primauté du libreéchange par rapport à toute politique commune. Certes, le référendum de 1975 sur le maintien britannique dans la Communauté Européenne ne s'était pas limité à proposer aux électeurs l'adhésion à un marché. II se référait aussi spécifiquement à des valeurs et but communs: bâtir une union des peuples, améliorer le niveau de vie, et maintenir la paix en Europe. Mais l'idée, centrale pour l'Allemagne et la France, de communauté de destin, d'une intégration de l'Europe dont l'ambition ne se limiterait pas à la seule création d'un marché, n'avait guère de place dans les politiques européennes de la plupart des gouvernements qui suivirent celui de Harold Wilson. Abondant dans le sens de François Mitterrand, l'ancien Chancelier Helmut Schmidt connaisseur du monde anglo-saxon, exprimait crûment des vues pessimistes sur l'insularité des sujets de Sa Majesté: "Il serait bien illusoire de considérer l'Angleterre comme un partenaire potentiel (pour remplacer le partenariat avec la France). L'inclination fondamentalement insulaire des Britanniques, tournés vers l'Atlantique, continuera d'entraver tous les gouvernements de Londres pendant plusieurs décennies, présentant l'alliance britannique avec les Etats-Unis comme considérablement plus importante que l'Union européenne." ${ }^{4}$ Des différences fondamentales donnaient ainsi tout son crédit à l'analyse politique de Bismarck, même si celle-ci fut rédigée dans un autre contexte et face à une configuration de puissances différente. La référence aux "deux" pouvait ainsi s'appliquer à l'entente franco-allemande, tandis que la puissance exclue, ou qui faisait librement un tel choix, était habituellement le Royaume-Uni.

Les sérieuses réserves britanniques face à une relation franco-allemande généralement perçue par Londres comme trop exclusive n'ont cependant pas empêché tantôt la France, tantôt l'Allemagne, de flirter parfois avec la tentation d'établir une relation spéciale entre Londres et leur capitale, qui viendrait contrebalancer le couple franco-allemand. Ce fut notamment le cas pour le Chancelier Gerhard Schröder ou les Présidents Chirac et Sarkozy, du moins dans les premiers temps de leur prise de fonction, jusqu'à ce que pour eux s'impose le constat de l'irremplaçabilité de l'entente franco-allemande. Ainsi, le RoyaumeUni pouvait-il remplir un rôle d'alternative politique, bien qu'improbable, dans une relation franco-allemande souvent complexe et difficile face aux grands défis européens. Sur le plan économique, l'orientation britannique vers une économie libre de marché faisait contrepoids face à une France qui, même passé le tournant des années 1990, faisait encore état de penchants économiques dirigistes, tout en lui rendant l'ordolibéralisme allemand plus fréquentable que la variété de capitalisme prônée par Londres (Trouille 2014; Uterwedde 2018). Néanmoins, la sortie prochaine du Royaume-Uni de l'UE remet en question ce type d'équilibres dans le jeu complexe entre les trois principales puissances européennes. II est certes probable que le Royaume-Uni post-Brexit restera arrimé à l'Europe dans de nombreux domaines dont dépend notamment la sécurité européenne. Redéfinir cette relation après le divorce du Brexit prendra du temps, des efforts et de la diplomatie. Mais il n'est pas exclu que cette redéfinition puisse clarifier une relation politique triangulaire jusqu'alors complexe et trop souvent difficile et déboucher sur une relation plus assainie.

\section{Montée du nationalisme anglais}

\footnotetext{
${ }^{4}$ Die Zeit, 14 octobre 1999, p.6.
} 
Deux ans après le second référendum qui s'est tenu le 23 juin 2016 sur le maintien du Royaume-Uni dans I'UE, pour nombre d'observateurs extérieurs, le Brexit demeure un phénomène largement incompréhensible. Comment un pays qui contribua si activement à façonner le Marché intérieur, et qui de surcroît bénéficie tant de son adhésion à l'Union, pouvait-il sérieusement considérer un tel divorce, avec toutes ses implications, économiques aussi bien que financières, commerciales, juridiques, sociales, géopolitiques, environnementales, médicales, universitaires ou scientifiques? Le Brexit représente une remise en cause fondamentale de tous ces référentiels, qui ont jusquà présent défini la place du Royaume-Uni en Europe et déterminé son importance dans le monde, comme pays-clé des décisions européennes, et comme porte ouverte aux investisseurs internationaux sur le Marché intérieur.

Car nul ne doit s'y tromper. Le Brexit est avant tout le projet d'une élite ultra-conservatrice et anti-européenne, mené par la droite dure du Parti Tory dans un contexte de renaissance d'un nationalisme non pas britannique, mais spécifiquement anglais, et soutenu par le leader du principal parti d'opposition, le travailliste Jeremy Corbyn. Ce projet est de surcroît défendu bec et ongles par une très large partie de la presse britannique, elle-même souvent contrôlée par cette élite qui, à l'instar de Rupert Murdoch et des frères Barclay, opère de l'étranger et réside souvent dans des paradis fiscaux. Leurs tabloïdes, viscéralement antieuropéens, matraquent quotidiennement et depuis de nombreuses années l'opinion publique de gros titres hostiles à "Bruxelles". Même la BBC, institution publique longtemps considérée comme hautement respectable et indépendante, ne transmet que des informations filtrées et manipulées sur toute question relative au Brexit, et accorde un temps de parole totalement disproportionné à ses partisans (Macshane 2017).

De plus, les conditions dans lesquelles le référendum fut organisé ont mis en évidence de très nombreuses irrégularités qui posent la question de sa légitimité juridique et démocratique (Auer 2017), amenant le philosophe britannique Anthony Grayling à comparer le Brexit à un coup politique préparé de longue date (Grayling 2017). Le référendum fut en effet présenté au Parlement britannique comme à caractère purement consultatif et non obligatoire. L'électorat fut restreint par élimination des catégories les plus concernées par un Brexit: résidents européens au Royaume-Uni, citoyens britanniques résidant à l'étranger depuis un certain nombre d'années, et jeunes générations de 16 et 17 ans, qui pourtant avaient pu voter lors du référendum sur l'indépendance de l'Ecosse. Les ressortissants de pays du Commonwealth, souvent moins concernés par la question, eurent la possibilité de voter. Des poursuites sont menées sur des sources avérées de finencement occulte de la campagne. De sérieuses présomptions pèsent sur une ingérence russe dans le résultat du référendum, que le gouvernement britannique a jusqu'à présent refusé d'enquêter. II a été démontré que des millions de profils facebook identifiés par les algorythmes de la compagnie d'analyse de données Cambridge Analytica comme susceptibles d'être influencés dans leur choix de vote ont été utilisés à cette fin. Une enquête est actuellement poursuivie à l'encontre de l'homme d'affaires Arron Banks par l'Agence nationale britannique de Lutte contre la Criminalité, une agence spécialisée dans les délits graves et la criminalité organisée. L'enquête porte sur de multiples infractions présumées lors de la campagne référendaire et notamment sur la provenance des financements apportés en faveur du retrait britannique. Des tentatives du Gouvernement de circonvenir les procédures parlementaires, notamment sur le déclenchement de l'article 50 du Traité de Lisbonne sur le retrait britannique, ont constitué une tentative d'accroître les prérogatives de l'exécutif au détriment du système parlementaire.

Les conditions souvent illégales dans lesquelles s'est déroulé le référendum, les mensonges qui ont mené à la victoire du retrait, les clivages internes au sein du gouvernement quant aux choix stratégiques à poursuivre dans les négociations avec l'UE, n'ont fait qu'aggraver 
des divisions déjà flagrantes au sein de la société britannique. D'une part en alimentant le populisme, d'autre part en créant un front pro-européen de résistance au Brexit qui rassembla près d'un million de personnes à Londres le 20 octobre 2018 pour exiger un vote populaire sur l'accord du Brexit alors en cours de négociation.

\section{Du libéralisme à l'illibéralisme: un déclin britannique annoncé}

Le vote référendaire du 23 juin 2016 a amorcé le déclin progressif du Royaume-Uni sur la scène internationale. Alors que ce pays était une puissance européenne majeure jouissant mondialement d'une grande influence au sein de l'UE, le Brexit change radicalement la donne. II représente une menace existentielle pour un pays qui se retranche sur lui-même. Projet fondamentalement populiste et nationaliste, le Brexit n'est guère plus que la version britannique de ce que représentent l'élection de Donald Trump aux Etats-Unis, Viktor Orban en Hongrie, la coalition gouvernementale en Italie, et d'autres formes de populisme à travers le monde. II repose sur un narratif émotionnel souverainiste et anti-européen qui ne se soucie guère d'exactitude, mais s'est avéré redoutablement efficace lors du référendum, notamment dans les riches campagnes conservatrices du sud et les régions postindustrielles délaissées du nord de l'Angleterre.

Suite au référendum, lors du Congrès annuel du Parti conservateur d'octobre 2016 à Brighton, l'aile libérale du parti, qui avait notamment compté comme figures de proue Margaret Thatcher, et plus récemment, jusqu'en 2016, David Cameron et son ministre des finances George Osborne, fut clairement supplantée par la droite souverainiste. Ce virage illibéral, protectionniste et interventionniste, marque ni plus ni moins l'échec du modèle anglo-saxon de développement. Les causes profondes de cet échec d'un libéralisme financier basé sur la shareholder value sont liées à l'accroissement exponentiel des inégalités, ainsi que le démontrent les travaux de l'économiste Thomas Piketti (Piketti 2013). L'austérité imposée par le gouvernement de David Cameron depuis la crise des subprimes, et un système de protection sociale devenu de plus en plus inefficace, avaient miné les couches sociales les plus défavorisées et accru les inégalités au sein de la société britannique. Certes, le phénomène est mondial. Néanmoins, l'échec du modèle libéral anglosaxon tel que celui-ci est pratiqué au Royaume-Uni n'augure pas la réussite de son contraire, le modèle souverainiste. Dans un monde de plus en plus interconnecté et interdépendant, où chaque pays tend naturellement à s'intégrer dans sa zone économique régionale, jouer cavalier seul requiert une prise de risque extrêmement audacieuse, sans commune mesure avec le poids économique, financier et militaire d'une puissance de taille moyenne. Le slogan de campagne appelant à "reprendre contrôle", ou celui lancé par Theresa May de "Global Britain", cache la réalité des interdépendances liées à tout engagement international, commercial ou autre. Pour Pascal Lamy, ancient directeur de l'Organisation mondiale du Commerce, le Brexit revient à tenter de reconstituer un oeuf à partir d'une omelette ${ }^{5}$.

Le grand paradoxe du Brexit est que "Global Britain" conduit non pas à une ouverture sur le monde, mais à un repli sur soi. Le Brexit signifie que le Royaume-Uni devient le seul pays au monde à s'imposer à lui-même des sanctions économiques. S'il se retire non seulement de l'UE, mais aussi du Marché intérieur et de l'Union douanière, il sera le seul pays au monde à tenter délibérément de renégocier avec tous ses partenaires commerciaux, d'Europe et d'ailleurs, des conditions commerciales moins favorables que celles dont il a jusqu'alors bénéficié. Extirper le pays de 45 années de traités européens et de réglementations communautaires, et renégocier systématiquement tous les arrangements commerciaux dont il bénéficie avec le reste du monde, est une gageure qui ne peut s'opérer

\footnotetext{
${ }^{5}$ Interview de Pascal Lamy sur France 24 le 15 janvier 2018.
} 
que par une réorientation radicale et généralisée de l'économie britannique dans quasiment tous les domaines d'activité. Plus le Brexit sera "dur", plus le Royaume-Uni pourrait s'orienter vers une économie de laisser-faire, à faible niveau d'imposition fiscale sur les sociétés, de faibles réglementations en matière sociale, environnementale, de sûreté alimentaire, phytosanitaire ou autre, seules conditions susceptibles pour son marché de préserver quelque attractivité et d'attirer capitaux et investissements internationaux. La perspective d'une telle mutation, qui ferait du Royaume-Uni un large paradis fiscal, une sorte de Singapour ou de Hong-Kong aux portes de l'UE ((Dunt, 2018), qui s'accompagnerait d'une forte dérégulation en matière de standards industriels, sociaux, sanitaires ou environnementaux, et d'une concurrence fiscale par le bas, enclin à user de pratiques économiques non coopératives et de réglementations protectionnistes en matière d'aides d'Etat, représente pour la France, l'Allemagne et l'Europe une sérieuse source d'inquiétudes qui expliquent la position adoptee par l'UE dans les négociations avec Londres d'aider sans rien lâcher.

\section{Impact du Brexit sur le couple franco-allemand - Rééquilibrage, ou nouveaux défis?}

Pour être efficace, la coopération entre la France et l'Allemange doit remplir plusieurs conditions. En premier lieu, elle doit reposer sur leur complémentarité et leur volonté commune d'oeuvrer ensemble dans la recherche de consensus susceptibles de rassembler les Européens. Mais en second lieu, il lui faut aussi se baser sur un rapport de forces relativement équilibré et qui assure une certaine parité dans les prises de position communes des deux capitales. Or, le déséquilibre économique et commercial entre les deux principales économies continentales n'avait fait que s'agggraver depuis les années 1990 (Trouille 2012). Ce n'est que depuis 2017 que le Président Macron a lancé un programme de réformes, nécessaires à plus d'efficacité. Néanmoins, le fossé entre excédents commerciaux allemands et déficits français continuait de s'accroître: en 2018. L'Allemagne devrait dégager, pour la troisième année consécutive, le plus important excédent des comptes courants au monde, soit 262 milliards d'euros. ${ }^{6}$

Le Brexit n'inversera pas cette tendance, mais pourrait toutefois l'atténuer, par les changements qu'il entraîne, dans les rapports de forces au sein de l'UE, et dans ceux qu'entretiennent la France et l'Allemagne. La dépendance allemande de ses exportations pourrait pénaliser des branches importantes de son économie, pour lesquelles le marché britannique compte, tels le secteur automobile, et ainsi affecter ses comptes publics. Mais l'une des conséquences les plus directes du Brexit est qu'il modifie l'équilibre des forces entre Etats-membres. Si la France demeure le second pays d'Europe par la taille de son PIB et son poids démographique, elle cesse de partager cette position ex aequo avec le Royaume-Uni après son retrait qui voit les Britanniques se retirer de la table où sont prises les décisions européennes. L'Italie passe en troisième position, à parité économique avec l'Espagne dont le PIB est désormais comparable au sien, la Pologne prend la cinquième position, et ainsi de suite. La défection d'un acteur de poids sur la scène européenne tend donc à consolider les positions d'autres Etats membres plus larges que la moyenne, et par là même, leur influence.

Mais la principale redistribution des cartes concerne l'Allemagne et la France. Le retrait de l'un des poids-lourds européens signifie, non seulement, que les deux pays profitent en terms d'influence de la désaffection du troisième "grand", mais aussi que, ensemble, ils vont peser davantage sur la scène européenne, et bien sûr dans les prises de décisions du

\footnotetext{
${ }^{6}$ Source: IFO.
} 
Conseil. En effet, hormis le Royaume-Uni, la part combinée du Produit intérieur brut européen détenue par l'Allemagne et la France monte à $43 \%$. Leurs parts du commerce de I'UE montent jusqu'à $44 \%$,et les deux pays représentent ensemble $33 \%$ de sa population, ainsi que $49 \%$ des dépenses militaires européennes. Le poids économique et commercial franco-allemand en Europe augmente donc substantiellement en proportion par le simple effet du retrait britannique. Certes, ce retrait laisse aussi un vacuum en Europe sur le terrain du libéralisme économique, que les Etats membres du nord cherchent, non sans peine, à combler en recréant une alliance à l'image de l'ancienne Ligue hanséatique.

Toutefois, le Brexit tend à conforter la France dans plusieurs domaines où celle-ci dispose d'un avantage comparatif par rapport à l'Allemagne. II induit ainsi un rééquilibrage partiel face à la dominance allemande sur le plan économique. Ainsi, la sortie de l'UE du RoyaumeUni représente pour l'Europe une perte de $28 \%$ de son potentiel militaire. La France se retrouve seule puissance militaire de poids, et seule puissance nucléaire, dans le concert européen. De plus, le départ du Royaume-Uni signifie également que la France, de par son siège permanent au Conseil de Sécurité des Nations Unies, devient de fait la seule courroie de transmission de l'Europe au sein de cette instance. Sur les questions de défense et de profil international, la France devient donc de plus en plus incontournable. La coopération militaire franco-allemande et la nécessité de construire l'Europe de la défense ne peuvent que s'en trouver renforcées compte tenu de la volonté partagé de Paris et de Berlin d'avancer dans cette voie. L'appel d'Emmanuel Macron à une "autonomie stratégique" vis-àvis des Etats-Unis, visant à se doter des moyens de ne pas être dépendant d'un autre, est relayé par Heiko Maas, Ministre fédéral des affaires étrangères, appelant à "une Europe souveraine et forte" avec la France et les autres Etats membres, laissant entrevoir un terrain d'action franco-allemande sur lequel consolider l'Europe est devenu un impératif. ${ }^{7}$

Certes, ces rééquilibrages ne doivent pas faire oublier les conséquences économiques, commerciales, budgétaires ou financières du Brexit, qui se feront sentir dans de multiples domaines et secteurs d'activité à travers l'Europe, qu'il y ait ou non accord avec Bruxelles sur une sortie ordonnée du Royaume-Uni. Certains secteurs, comme l'industrie automobile, notamment en Allemagne, pourraient être sérieusement affectés par un Brexit "dur" sans accord. Toutefois, même si l'Allemagne est particulièrement vulnérable de par sa dépendance au commerce, c'est le commerce extérieur des trois pays comme celui d'autres partenaires européens qui se verra affecté. Les relocalisations industrielles et financières du Royaume-Uni vers le continent pourraient toutefois profiter davantage à la France qu'à l'Allemagne, et la force d'attraction de la place financière de Paris sur les banques de la City se trouve renforcée par la relocalisation de Londres vers la capitale française du siège de l'Autorité bancaire européenne. Paris pourrait ainsi se retrouver grande gagnante de la course à la relocalisation des activités bancaires de la City, devançant Francfort, Dublin et Amsterdam. ${ }^{8}$ Engagée sur un programme de réformes et un meilleur contrôle de ses déficits budgétaires, la France véhicule une nouvelle image et regagne en crédibilité aux yeux d'investisseurs étrangers.

Face aux multiples écueils que le retrait du Royaume-Uni laisse entrevoir, Berlin et Paris ont dans l'ensemble suivi une trajectoire identique dans le cadre des négociations entre Bruxelles et Londres, et ont été suivis par l'ensemble de leurs partenaires européens, qui ont fait preuve d'une cohesion tout aussi remarquable qu'inhabituelle. L'un des effets du Brexit aura été jusqu'à présent de renforcer la cohésion entre Etats membres, du moins sur l'importance de préserver les acquis européens, notamment le Marché intérieur. Certes, les Européens sont loin de parler d'une seule voix sur d'autres questions aussi fondamentales que les migrations, ni d'avoir des vues convergentes sur les moyens de consolider la zone euro ou de bâtir une défense européenne, difficile à envisager sans l'affirmation d'une

\footnotetext{
${ }^{7}$ Handelsblatt, 22 août 2018.

${ }^{8}$ Financial Times, 1 octobre 2018.
} 
Europe politique. Mais elle compte face aux menaces qui pèsent sur l'intégration européenne. II était essentiel que I'UE, et en premier lieu l'Allemagne et la France, se montrent unis et vigilants dans les négociations de sortie de l'Union. Face à un RoyaumeUni prompt à réclamer un accord hors du Marché intérieur et de l'Union douanière, mais cherchant à s'attribuer un maximum d'avantages de ces derniers aux dépens du contribuable européen, sans en accepter les disciplines ni contribuer au budget européen et en rejetant la liberté de movement, il était necessaire de faire front. Angela Merkel a parfois pu donner l'impression de se montrer plus flexible, tandis qu'Emmanuel Macron se sera montré plus intransigeant. Mais il ne s'agit là que de nuances d'une position francoallemande unie visant à aider mais sans rien concéder sur les principes.

\section{Conclusions}

A quels défis l'Allemagne et la France vont-elles devoir faire face dans une Europe postBrexit? En dépit de sa nocivité pour le projet européen et de ses sérieuses implications économiques, financières et commerciales, le Brexit n'est que l'une des facettes de l'incroyable défi que doivent affronter ensemble les deux pays, face à un monde extérieur chaque jour plus hostile, comme au sein de l'Europe et dans leurs propres frontières nationales. Ce défi omniprésent de la poussée nationaliste et de marchands d'illusion populistes constitue l'un des plus graves dangers auxquels l'Europe est confrontée. La montée des populismes dans la presque totalité des démocraties occidentales, leur installation au pouvoir, l'augmentation rapide du nombre de régimes illibéraux, la désaffection électorale à l'égard des deux grandes formations politiques traditionnelles, social-démocratie et démocratie chrétienne, la politisation à outrance de la question migratoire, constituent aujourd'hui une nouvelle donne, un changement d'époque au moins aussi fondamental que le fut la chute du mur de Berlin le 9 novembre 1989. L'alliance atlantique, sur laquelle était fondée la stabilité internationale d'après-guerre, est aujourd'hui fracturée. Les fondamentaux que constituent le multilatéralisme, l'aspiration au libreéchange, l'unité européenne, et l'opprobe culturelle sur le racisme, les extrême-droites et les nationalismes, sont remis en cause. Dès mai 2017, Angela Merkel en établissait le constat, déclarant: "Nous, les Européens, devons prendre notre destin en main". ${ }^{9}$

Mais la volonté est-elle encore au rendez-vous? Angela Merkel et Emmanuel Macron auraient-ils manqué leur moment européen? Quel leadership une Allemagne en situation de crise politique peut-elle offrir à l'Europe? Tous deux prônent l'établissement d'"une vraie armée européenne", mais quels compromis seront nécessaires pour y parvenir? La redistribution des cartes sur l'échiquier politique allemand, l'entrée en force du parti antimigrants AfD au Bundestag ainsi que dans plusieurs parlements régionaux, le recul des deux grands partis politiques traditionnels, ne remettront-ils pas en question la survie de la grande coalition? La chancelière Merkel ne se verra-t-elle pas contrainte de préserver ce qui peut l'être plutôt que de se lancer dans de grands chantiers, comme la poursuite du dialogue franco-allemand sur la consolidation de l'eurozone ou l'élaboration de cette défense européenne indispensable, que la France avait trop hâtivement rejetée en 1954? Tandis que le fossé se creuse en Allemagne entre économie et politique, la fragmentation du paysage politique et sa recomposition rendent de plus en plus complexe la formation de coalitions et la poursuite de projets ambitieux de réforme en Europe. Une Allemagne politiquement frileuse et divisée mais d'une potence économique rare, et une France engagée dans une

\footnotetext{
${ }^{9}$ Le Monde, 23/08/2018
} 
longue convalescence mais politiquement et militairement incontrournable, doivent compter sur leur complémentarité et une determination sans faille pour exercer un leadership sans concessions à l'égard des courants nationalistes.

Pourtant, ces projets de consolidation sont plus que jamais nécessaires. Plus les défis européens s'accumulent, et plus l'Europe paraît fragile. A l'heure où l'UE, géant économique mais nain politique et militaire, est de plus en plus remise en cause par les autres grandes puissances, où la Chine s'apprête à devenir la principale puissance économique et deuxième puissance militaire au monde, où la gouvernance globale est en crise, où la technologie n'est plus une chasse gardée de l'occident, où la démographie occidentale recule tandis que la population africaine est en passe de devenir dans les trente ans la plus nombreuse de toutes, où les occidentaux s'offrent le luxe de se diviser, où le Brexit ampute le projet européen d'un Etat membre certes difficile, mais essentiel, les Européens, plus que jamais, se doivent de prendre leur destin collectif en mains. Alors que l'intégration européenne se voit ébranlée par l'instabilité croissante du monde extérieur et la montée des nationalismes de toutes parts comme au sein même de l'UE, l'entente franco-allemande, construite sur les ruines du fascisme pour garantir la paix en Europe, garde plus que jamais tout son sens.Il lui faut mener ce combat essentiel avant que l'Europe ne cesse de compter dans le monde.

\section{Bibliographie}

Auer, C. (2017) Le référendum britannique du 23 juin 2016: un simulacra de démocratie? Revue française de civilisation britannique (en ligne) XXII-1 2017.

Dunt, I. (2018) Brexit - What the Hell Happens Now? Canbury Press, Surrey.

Grayling, A. C. (2017) Democracy and its Crisis. Oneworld, London.

Maclean, M. et Trouille, J-M. (2001) France, Germany and Britain - Partners in a Changing World, Palgrave, Hampshire.

Macshane, (2017) D. Brexit, No Exit - Why (in the end) Britain won't Leave Europe. I.B. Tauris, Croydon.

Mitterrand, F. (1996) De l'Allemagne, de la France, Odile Jacob, Paris.

Piketti, T. (2013) Le capital au XXle siècle, Seuil, Paris.

Thatcher, M. (1995) Les Chemins du pouvoir, Mémoires II, Albin Michel.

Trouille, J-M. (2012) L'importance de l'économie et de l'industrie dans les relations bilatérales franco-allemandes - Interdépendance, convergence des politiques, ou décrochage? Paru dans: "Les relations franco-allemandes dans une Europe unifiée Réalisations et défis", sous la direction de Hans Stark, Martin Koopman et Joachim Schild, Presses universitaires de Bordeaux. 
Trouille, J-M. (2014) 'Industrial Nationalism versus European Partnerships: An Analysis of State-led Franco-German Inter-Firm Linkages', Environment and Planning C: Government and Policy, 32(6), 1059-82.

Uterwedde, H. (2017) Frankreich, eine Länderkunde, Verlag Barbara Budrig, Opladen, Berlin, Toronto. 